\title{
Erratum to: Dynamic flight stability of hovering model insects: theory versus simulation using equations of motion coupled with Navier-Stokes equations
}

\author{
Yan Lai Zhang • Mao Sun
}

(C)The Chinese Society of Theoretical and Applied Mechanics and SpringerVerlag Berlin Heidelberg 2011

Erratum to: Acta Mechanica Sinica (2010) 26(4): 509-520

DOI: $10.1007 / \mathbf{s 1 0 4 0 9 - 0 1 0 - 0 3 6 0 - 5}$

The nondimensional variable " $q \mathrm{~b} / U$ " in Figs. $3 \mathrm{c}-12 \mathrm{c}$ should be " $q_{\mathrm{b}} c / U$ ".

The online version of the original article can be found at http://dx.doi.org/10.1007/s1040901003605.

Y.Ł. Zhang · M. Sun (区)

MinistryefEducation Key Laboratory of Fluid Mechanics,

Institute of Fluid Mechanics,

Beihang University, 100191 Beijing, China

email: m.sun@263.net 